J. Korean Math. Soc. 47 (2010), No. 3, pp. 573-583

DOI 10.4134/JKMS.2010.47.3.573

\title{
ANTI-PERIODIC SOLUTIONS FOR HIGHER-ORDER NONLINEAR ORDINARY DIFFERENTIAL EQUATIONS
}

\author{
Tai Yong Chen, Wen Bin Liu, Jian Jun Zhang, and Hui Xing Zhang
}

ABSTRACT. In this paper, the existence of anti-periodic solutions for higher-order nonlinear ordinary differential equations is studied by using degree theory and some known results are improved to some extent.

\section{Introduction}

Anti-periodic problems arise naturally from the mathematical models of a variety of physical processes and have important applications in auto-control, partial differential equations and engineering. Recently, there has been a great deal of research on anti-periodic boundary value problem (see [1], [2], [9], [10], [11], [12] and references therein). In mechanics, the simplest model of oscillation equation is single pendulum equation

$$
x^{\prime \prime}+\omega^{2} \sin x=p(t)=p(t+2 \pi),
$$

whose anti-periodic solutions satisfy

$$
x(t+\pi)=-x(t), \quad \forall t \in \mathbb{R} .
$$

In particular, many authors have discussed the existence of anti-periodic solutions for the first order or second order nonlinear ordinary differential equation. Mawhin ([9]) generalized equation (1) to the general Duffing equation

$$
x^{\prime \prime}+g(x)=p(t),
$$

and obtained the existence results for anti-periodic solutions by using critical point theory. In [1], the author proved the existence of anti-periodic solutions for the following abstract nonlinear second order evolution equation

$$
-x^{\prime \prime}(t)+a x^{\prime}(t)+A(t) x(t)=f(t)
$$

Received August 30, 2008.

2000 Mathematics Subject Classification. 34B15, 34C25.

Key words and phrases. higher-order differential equation, anti-periodic solution, LeraySchauder principle.

This work was financially supported by National Natural Science Foundation of China (10771212) and Science Foundation of China University of Mining and Technology (2008A037). 
associated with maximal monotone operators in Hilbert spaces. In [2], some results of anti-periodic solutions for Liénard equation

$$
x^{\prime \prime}+f(x) x^{\prime}+g(t, x)=p(t)
$$

were established by Leray-Schauder principle.

In recent years, many results relative to the existence of periodic solutions for higher-order ordinary differential equations have been obtained (see [3], [6], [7], [8] and references therein). In this paper, we consider the existence of antiperiodic solutions for the following higher-order nonlinear ordinary differential equations

$$
\begin{aligned}
& x^{(2 m)}+\sum_{i=2}^{2 m-1} a_{i} x^{(i)}+f(x) x^{\prime}+g(t, x)=p(t), \\
& x^{(2 m+1)}+\sum_{i=2}^{2 m} a_{i} x^{(i)}+f(x) x^{\prime}+g(t, x)=p(t),
\end{aligned}
$$

where $a_{i} \in \mathbb{R}(i=2,3, \ldots, 2 m), f(x) \in C(\mathbb{R}, \mathbb{R}), g(t, x) \in C\left(\mathbb{R}^{2}, \mathbb{R}\right)$ and $g(t+$ $2 \pi, x)=g(t, x), p(t) \in C(\mathbb{R}, \mathbb{R})$ and $p(t+2 \pi)=p(t)$. We obtain several useful results by using Leray-Schauder principle.

The plan of this paper is as follows. Section 2 contains the necessary preliminaries. In section 3, we obtain the existence theorem of anti-periodic solutions for equation (2) (Theorem 3.1). Section 4 consists of two parts. In the first part, we establish two existence theorems of anti-periodic solutions for equation (3) (Theorem 4.1, 4.2). The second part is devoted to handling with the equation (3) when $g(t, x)=g(x)$ and we obtain two existence results of antiperiodic solutions (Theorem 4.3,4.4). Our results improve and generalize some known results to some extent.

\section{Preliminaries}

Throughout the paper, we shall use the following notations

$$
\begin{aligned}
& C^{k, \pi}=\left\{x \in C^{k}(\mathbb{R}, \mathbb{R}): x(t+\pi)=-x(t), \forall t \in \mathbb{R}\right\}, \\
& \|x\|_{2}=\left\{\int_{0}^{2 \pi}|x(t)|^{2} d t\right\}^{1 / 2}, \quad\|x\|_{\infty}=\max _{t \in[0,2 \pi]}|x(t)|, \\
& \|x\|_{C^{k}}=\max _{i=0,1, \ldots, k}\left\{\left\|x^{(i)}\right\|_{\infty}\right\} .
\end{aligned}
$$

For $x(t) \in C^{0, \pi}$, there exists the following Fourier expansion

$$
x(t)=\sum_{i=0}^{\infty}\left[a_{2 i+1} \cos (2 i+1) t+b_{2 i+1} \sin (2 i+1) t\right] .
$$


Let us define $J: C^{0, \pi} \longrightarrow C^{1, \pi}$

$$
\begin{aligned}
(J x)(t) & =\int_{0}^{t} x(s) d s-\sum_{i=0}^{\infty} \frac{b_{2 i+1}}{2 i+1} \\
& =\sum_{i=0}^{\infty}\left[\frac{a_{2 i+1}}{2 i+1} \sin (2 i+1) t-\frac{b_{2 i+1}}{2 i+1} \cos (2 i+1) t\right] .
\end{aligned}
$$

Obviously

By the definition of $J$, we have

$$
\frac{d}{d t}[J x(t)]=x(t)
$$

$$
\begin{aligned}
|(J x)(t)| & \leq \int_{0}^{2 \pi}|x(s)| d s+\sum_{i=0}^{\infty} \frac{\left|b_{2 i+1}\right|}{2 i+1} \\
& \leq 2 \pi\|x\|_{\infty}+\left(\sum_{i=0}^{\infty} b_{2 i+1}^{2}\right)^{1 / 2}\left[\sum_{i=0}^{\infty} \frac{1}{(2 i+1)^{2}}\right]^{1 / 2}
\end{aligned}
$$

Noting

$$
\left[\sum_{i=0}^{\infty} \frac{1}{(2 i+1)^{2}}\right]^{1 / 2}=\frac{\pi}{2 \sqrt{2}}
$$

and using the Parseval equality

$$
\int_{0}^{2 \pi}|x(s)|^{2} d s=\pi \sum_{i=0}^{\infty}\left(a_{2 i+1}^{2}+b_{2 i+1}^{2}\right)
$$

we obtain

Immediately

$$
\begin{aligned}
|(J x)(t)| & \leq 2 \pi\|x\|_{\infty}+\frac{\pi}{2 \sqrt{2}}\left[\sum_{i=0}^{\infty}\left(a_{2 i+1}^{2}+b_{2 i+1}^{2}\right)\right]^{1 / 2} \\
& =2 \pi\|x\|_{\infty}+\frac{\pi}{2 \sqrt{2}}\left(\frac{1}{\pi} \int_{0}^{2 \pi}|x(s)|^{2} d s\right)^{1 / 2} \\
& \leq 2 \pi\|x\|_{\infty}+\frac{\pi}{2}\|x\|_{\infty}=\frac{5 \pi}{2}\|x\|_{\infty}, \quad \forall t \in[0,1] .
\end{aligned}
$$

$$
\|J x\|_{\infty} \leq \frac{5 \pi}{2}\|x\|_{\infty} .
$$

Therefore, $J$ is continuous. It is easy to prove that $J$ is a completely continuous operator by Arzela-Ascoli theorem.

Moreover, we will need the following lemmas.

Lemma $2.1([5])$. Assume that $x(t) \in C^{1}(\mathbb{R}, \mathbb{R})$ and $x(0)=x(2 \pi), \int_{0}^{2 \pi} x(t) d t=$ 0 , then

$$
\int_{0}^{2 \pi}|x(t)|^{2} d t \leq \int_{0}^{2 \pi}\left|x^{\prime}(t)\right|^{2} d t .
$$


Lemma $2.2([4])$. Suppose $\Omega$ is a bounded open set of normal space $X, f$ is compact in $\bar{\Omega}$ and $p \in X \backslash f(\partial \Omega)$. Then the equation $f(x)=p$ has a solution in $\Omega$, provided with $\operatorname{deg}(f, \Omega, p) \neq 0$.

\section{The existence of anti-periodic solutions for equation (2)}

In this section, we will prove the existence of anti-periodic solutions for equation (2).

Theorem 3.1. Assume that

$\left(H_{1}\right)$ for $t \in \mathbb{R}, x \in \mathbb{R}$

$$
f(-x)=f(x), \quad g(t+\pi,-x)=-g(t, x), \quad p(t+\pi)=-p(t) ;
$$

$\left(H_{2}\right)$ there is $\alpha \geq 0$ such that

$$
\limsup _{|x| \rightarrow+\infty} \frac{|g(t, x)|}{|x|}=\alpha, \quad \forall t \in \mathbb{R}
$$

$\left(H_{3}\right) 1-\sum_{i=1}^{m-1}\left|a_{2 i}\right|-\alpha>0$.

Then there exists at least one anti-periodic solution of equation (2).

Proof. We consider the auxiliary equation of (2)

$$
\begin{aligned}
x^{(2 m)} & =-\lambda \sum_{i=2}^{2 m-1} a_{i} x^{(i)}-\lambda f(x) x^{\prime}-\lambda g(t, x)+\lambda p(t) \\
& :=\lambda q_{1}\left(x^{(2 m-1)}, \ldots, x^{\prime}, x, t\right),
\end{aligned}
$$

where $\lambda \in[0,1]$. Obviously, $q_{1}\left(x^{(2 m-1)}, \ldots, x^{\prime}, x, t\right)$ is continuous.

Firstly, we can claim that there exists a prior bound in $C^{2 m, \pi}$, for the possible solution $x(t)$ of equation (4).

Multiplying equation (4) with $x(t)$ and integrating it over $[0,2 \pi]$, we get

$$
\begin{aligned}
& \int_{0}^{2 \pi} x^{(2 m)}(t) x(t) d t \\
= & -\lambda \sum_{i=2}^{2 m-1} a_{i} \int_{0}^{2 \pi} x^{(i)}(t) x(t) d t-\lambda \int_{0}^{2 \pi} f(x(t)) x^{\prime}(t) x(t) d t \\
& -\lambda \int_{0}^{2 \pi} g(t, x(t)) x(t) d t+\lambda \int_{0}^{2 \pi} p(t) x(t) d t .
\end{aligned}
$$

Noting $\int_{0}^{2 \pi} x^{(2 i+1)}(t) x(t) d t=0$ and

$$
\int_{0}^{2 \pi} f(x(t)) x^{\prime}(t) x(t) d t=\int_{0}^{2 \pi} f(x(t)) x(t) d(x(t))=\int_{x(0)}^{x(2 \pi)} f(\tau) \tau d \tau=0
$$


combining with $\int_{0}^{2 \pi} x^{(2 m)}(t) x(t) d t=(-1)^{m} \int_{0}^{2 \pi}\left|x^{(m)}(t)\right|^{2} d t$, we have

$$
\begin{aligned}
& \int_{0}^{2 \pi}\left|x^{(m)}(t)\right|^{2} d t \\
= & (-1)^{m+1} \lambda \sum_{i=1}^{m-1} a_{2 i} \int_{0}^{2 \pi} x^{(2 i)}(t) x(t) d t+(-1)^{m+1} \lambda \int_{0}^{2 \pi} g(t, x(t)) x(t) d t \\
& +(-1)^{m} \lambda \int_{0}^{2 \pi} p(t) x(t) d t \\
\leq & \sum_{i=1}^{m-1}\left|a_{2 i}\right|\left|\int_{0}^{2 \pi} x^{(2 i)}(t) x(t) d t\right|+\int_{0}^{2 \pi}|g(t, x(t)) x(t)| d t \\
& +\int_{0}^{2 \pi}|p(t) x(t)| d t .
\end{aligned}
$$

By hypothesis $\left(H_{2}\right)$, we can find some constant $\beta \geq 0$ such that

$$
|g(t, x)| \leq \beta+\alpha|x|, \quad \forall t, x \in \mathbb{R} .
$$

Thus

$$
\begin{aligned}
& \int_{0}^{2 \pi}\left|x^{(m)}(t)\right|^{2} d t \\
\leq & \sum_{i=1}^{m-1}\left|a_{2 i}\right| \int_{0}^{2 \pi}\left|x^{(i)}(t)\right|^{2} d t+\int_{0}^{2 \pi}(\beta+\alpha|x(t)|)|x(t)| d t \\
& +\int_{0}^{2 \pi}|p(t) x(t)| d t \\
\leq & \sum_{i=1}^{m-1}\left|a_{2 i}\right|\left\|x^{(i)}\right\|_{2}^{2}+\alpha\|x\|_{2}^{2}+\left(\sqrt{2 \pi} \beta+\|p\|_{2}\right)\|x\|_{2} .
\end{aligned}
$$

For $x(t) \in C^{2 m, \pi}$, we get $\int_{0}^{2 \pi} x^{(i)}(t) d t=0(i=0,1, \ldots, 2 m-1)$. By Lemma 2.1 , it can be shown that

$$
\|x\|_{2} \leq\left\|x^{\prime}\right\|_{2} \leq \cdots \leq\left\|x^{(2 m)}\right\|_{2} .
$$

So, from (5)

$$
\left\|x^{(m)}\right\|_{2}^{2} \leq \sum_{i=1}^{m-1}\left|a_{2 i}\right|\left\|x^{(m)}\right\|_{2}^{2}+\alpha\left\|x^{(m)}\right\|_{2}^{2}+\left(\sqrt{2 \pi} \beta+\|p\|_{2}\right)\left\|x^{(m)}\right\|_{2} .
$$

By assumption $\left(H_{3}\right)$, there exists $M_{1}>0$ (independent of $\lambda$ ) such that

$$
\|x\|_{2} \leq\left\|x^{\prime}\right\|_{2} \leq \cdots \leq\left\|x^{(m)}\right\|_{2} \leq M_{1} .
$$


Because $\int_{0}^{2 \pi} x(t) d t=0$, there exists $t_{0} \in[0,2 \pi]$ such that $x\left(t_{0}\right)=0$. Hence

$$
\|x\|_{\infty} \leq \int_{0}^{2 \pi}\left|x^{\prime}(t)\right| d t \leq \sqrt{2 \pi}\left\|x^{\prime}\right\|_{2} \leq \sqrt{2 \pi} M_{1} .
$$

A similar argument, we can prove

$$
\left\|x^{(i)}\right\|_{\infty} \leq \sqrt{2 \pi} M_{1}, \quad i=1,2, \ldots, m-1 .
$$

Multiplying equation (4) with $x^{(2 m)}(t)$ and integrating it over $[0,2 \pi]$, we get

$$
\begin{aligned}
& \int_{0}^{2 \pi}\left|x^{(2 m)}(t)\right|^{2} d t \\
= & -\lambda \sum_{i=2}^{2 m-1} a_{i} \int_{0}^{2 \pi} x^{(i)}(t) x^{(2 m)}(t) d t-\lambda \int_{0}^{2 \pi} f(x(t)) x^{\prime}(t) x^{(2 m)}(t) d t \\
& -\lambda \int_{0}^{2 \pi} g(t, x(t)) x^{(2 m)}(t) d t+\lambda \int_{0}^{2 \pi} p(t) x^{(2 m)}(t) d t \\
\leq & \sum_{i=2}^{2 m-1}\left|a_{i}\right|\left|\int_{0}^{2 \pi} x^{(i)}(t) x^{(2 m)}(t) d t\right|+\int_{0}^{2 \pi}\left|f(x(t)) x^{\prime}(t) x^{(2 m)}(t)\right| d t \\
& +\int_{0}^{2 \pi}\left|g(t, x(t)) x^{(2 m)}(t)\right| d t+\int_{0}^{2 \pi}\left|p(t) x^{(2 m)}(t)\right| d t .
\end{aligned}
$$

For $\int_{0}^{2 \pi} x^{(2 i+1)}(t) x^{(2 m)}(t) d t=0$, we can see

$$
\begin{aligned}
& \int_{0}^{2 \pi}\left|x^{(2 m)}(t)\right|^{2} d t \\
\leq & \sum_{i=1}^{m-1}\left|a_{2 i}\right|\left|\int_{0}^{2 \pi} x^{(2 i)}(t) x^{(2 m)}(t) d t\right|+\int_{0}^{2 \pi}\left|f(x(t)) x^{\prime}(t) x^{(2 m)}(t)\right| d t \\
& +\int_{0}^{2 \pi}\left|g(t, x(t)) x^{(2 m)}(t)\right| d t+\int_{0}^{2 \pi}\left|p(t) x^{(2 m)}(t)\right| d t .
\end{aligned}
$$

By (6), there exist $\gamma_{1}, \gamma_{2} \geq 0$ such that $|f(x)| \leq \gamma_{1},|g(t, x)| \leq \gamma_{2}, \forall t, x \in \mathbb{R}$. Hence, from (7) and (8), we can get

$$
\begin{aligned}
& \int_{0}^{2 \pi}\left|x^{(2 m)}(t)\right|^{2} d t \\
\leq & \sum_{i=1}^{m-1}\left|a_{2 i}\right| \int_{0}^{2 \pi}\left|x^{(i+m)}(t)\right|^{2} d t+\left(\sqrt{2 \pi} M_{1} \gamma_{1}+\gamma_{2}\right) \int_{0}^{2 \pi}\left|x^{(2 m)}(t)\right| d t \\
& +\int_{0}^{2 \pi}\left|p(t) x^{(2 m)}(t)\right| d t \\
\leq & \sum_{i=1}^{m-1}\left|a_{2 i}\right|\left\|x^{(i+m)}\right\|_{2}^{2}+\left(2 \pi M_{1} \gamma_{1}+\sqrt{2 \pi} \gamma_{2}+\|p\|_{2}\right)\left\|x^{(2 m)}\right\|_{2} .
\end{aligned}
$$


By using Lemma 2.1, we know

$$
\left\|x^{(2 m)}\right\|_{2}^{2} \leq \sum_{i=1}^{m-1}\left|a_{2 i}\right|\left\|x^{(2 m)}\right\|_{2}^{2}+\left(2 \pi M_{1} \gamma_{1}+\sqrt{2 \pi} \gamma_{2}+\|p\|_{2}\right)\left\|x^{(2 m)}\right\|_{2} .
$$

For $\left(H_{3}\right)$, there exists $M_{2}>0$ (independent of $\lambda$ ) such that

$$
\|x\|_{2} \leq\left\|x^{\prime}\right\|_{2} \leq \cdots \leq\left\|x^{(2 m)}\right\|_{2} \leq M_{2} \text {. }
$$

Similar with the proof of (6), we can prove

$$
\left\|x^{(i)}\right\|_{\infty} \leq \sqrt{2 \pi} M_{2}, \quad i=0,1, \ldots, 2 m-1
$$

By the equation (4), there exists $M_{3}>0$ (independent of $\lambda$ ) such that

$$
\left\|x^{(2 m)}\right\|_{\infty} \leq M_{3}
$$

Set $T_{1}=\max \left\{\sqrt{2 \pi} M_{2}, M_{3}\right\}+1$. Then

$$
\|x\|_{C^{2 m}}<T_{1} .
$$

Secondly, we can prove the existence of anti-periodic solutions for equation (2). Set

$$
\Omega=\left\{x(t) \in C^{2 m, \pi}:\|x\|_{C^{2 m}}<T_{1}\right\} .
$$

Then $\Omega$ is a bounded open set in $C^{2 m, \pi}$. By hypothesis $\left(H_{1}\right)$, it is easy to see that

$$
\begin{aligned}
& q_{1}\left(x^{(2 m-1)}(t+\pi), \ldots, x^{\prime}(t+\pi), x(t+\pi), t+\pi\right) \\
= & -q_{1}\left(x^{(2 m-1)}(t), \ldots, x^{\prime}(t), x(t), t\right), \quad \forall x(t) \in C^{2 m, \pi} .
\end{aligned}
$$

Hence $q_{1}: C^{2 m-1, \pi} \longrightarrow C^{0, \pi}$. Define $F_{\lambda}: \bar{\Omega} \longrightarrow C^{2 m, \pi}$

$$
F_{\lambda} x=\lambda J^{2 m} q_{1} x, \quad \lambda \in[0,1] .
$$

Obviously, $F_{\lambda}$ is compact. Hence, the fixed points of $F_{1}$ in $\bar{\Omega}$ are the antiperiodic solutions of equation (2).

Let $h_{\lambda}(x): \bar{\Omega} \times[0,1] \longrightarrow C^{2 m, \pi}$

$$
h_{\lambda}(x)=x-F_{\lambda} x .
$$

By $(9)$, we get $\theta \notin h_{\lambda}(\partial \Omega)$. Hence

$$
\begin{aligned}
\operatorname{deg}\left(i d-F_{1}, \Omega, \theta\right) & =\operatorname{deg}\left(h_{1}, \Omega, \theta\right)=\operatorname{deg}\left(h_{0}, \Omega, \theta\right) \\
& =\operatorname{deg}(i d, \Omega, \theta)=1 .
\end{aligned}
$$

Consequently, $F_{1}$ has at least one fixed point in $\Omega$ by Lemma 2.2. Namely, the equation (2) has at least one anti-periodic solution. 


\section{The existence of anti-periodic solutions for equation (3)}

In this section, we will prove the existence of anti-periodic solutions for equation (3).

Theorem 4.1. Assume that

$\left(H_{4}\right)$ for $x \in \mathbb{R}$

$$
(-1)^{m+1} f(x) \leq 0
$$

$\left(H_{5}\right) 1-\sum_{i=1}^{m-1}\left|a_{2 i+1}\right|-\alpha>0$

and the assumptions $\left(H_{1}\right),\left(H_{2}\right)$ are true. Then there exists at least one antiperiodic solution of equation (3).

Proof. We consider the auxiliary equation of (3)

$$
\begin{aligned}
x^{(2 m+1)} & =-\lambda \sum_{i=2}^{2 m} a_{i} x^{(i)}-\lambda f(x) x^{\prime}-\lambda g(t, x)+\lambda p(t) \\
& :=\lambda q_{2}\left(x^{(2 m)}, \ldots, x^{\prime}, x, t\right),
\end{aligned}
$$

where $\lambda \in[0,1]$. Obviously, $q_{2}\left(x^{(2 m)}, \ldots, x^{\prime}, x, t\right)$ is continuous.

Similar with the proof of Theorem 3.1, we need only prove that there exists a prior bound in $C^{2 m+1, \pi}$, for the possible solution $x(t)$ of equation (10).

Multiplying equation (10) with $x^{\prime}(t)$ and integrating it over $[0,2 \pi]$, we get

$$
\begin{aligned}
& \int_{0}^{2 \pi} x^{(2 m+1)}(t) x^{\prime}(t) d t \\
= & -\lambda \sum_{i=2}^{2 m} a_{i} \int_{0}^{2 \pi} x^{(i)}(t) x^{\prime}(t) d t-\lambda \int_{0}^{2 \pi} f(x(t))\left(x^{\prime}(t)\right)^{2} d t \\
& -\lambda \int_{0}^{2 \pi} g(t, x(t)) x^{\prime}(t) d t+\lambda \int_{0}^{2 \pi} p(t) x^{\prime}(t) d t .
\end{aligned}
$$

Noting $\int_{0}^{2 \pi} x^{(2 i)}(t) x^{\prime}(t) d t=0$ and

$$
\int_{0}^{2 \pi} x^{(2 m+1)}(t) x^{\prime}(t) d t=(-1)^{m} \int_{0}^{2 \pi}\left|x^{(m+1)}(t)\right|^{2} d t
$$

we have

$$
\begin{aligned}
& \int_{0}^{2 \pi}\left|x^{(m+1)}(t)\right|^{2} d t \\
= & (-1)^{m+1} \lambda \sum_{i=1}^{m-1} a_{2 i+1} \int_{0}^{2 \pi} x^{(2 i+1)}(t) x^{\prime}(t) d t \\
& +(-1)^{m+1} \lambda \int_{0}^{2 \pi} f(x(t))\left(x^{\prime}(t)\right)^{2} d t \\
& +(-1)^{m+1} \lambda \int_{0}^{2 \pi} g(t, x(t)) x^{\prime}(t) d t+(-1)^{m} \lambda \int_{0}^{2 \pi} p(t) x^{\prime}(t) d t .
\end{aligned}
$$


By the assumption $\left(H_{4}\right)$, we can see

$$
\begin{aligned}
& \int_{0}^{2 \pi}\left|x^{(m+1)}(t)\right|^{2} d t \\
\leq & \sum_{i=1}^{m-1}\left|a_{2 i+1}\right|\left|\int_{0}^{2 \pi} x^{(2 i+1)}(t) x^{\prime}(t) d t\right|+\int_{0}^{2 \pi}\left|g(t, x(t)) x^{\prime}(t)\right| d t \\
& +\int_{0}^{2 \pi}\left|p(t) x^{\prime}(t)\right| d t \\
\leq & \sum_{i=1}^{m-1}\left|a_{2 i+1}\right| \int_{0}^{2 \pi}\left|x^{(i+1)}(t)\right|^{2} d t+\int_{0}^{2 \pi}\left|g(t, x(t)) x^{\prime}(t)\right| d t \\
& +\int_{0}^{2 \pi}\left|p(t) x^{\prime}(t)\right| d t .
\end{aligned}
$$

A similar argument with Theorem 3.1, we can prove that there exists at least one anti-periodic solution of equation (3).

Similar with Theorem 4.1, we can obtain the following result.

Theorem 4.2. Assume that

$\left(H_{6}\right)$ there is $M \geq 0$ such that

$$
|f(x)| \leq M, \quad \forall x \in \mathbb{R}
$$

$\left(H_{7}\right) 1-\sum_{i=1}^{m-1}\left|a_{2 i+1}\right|-M-\alpha>0$

and the assumptions $\left(H_{1}\right),\left(H_{2}\right)$ are true. Then there exists at least one antiperiodic solution of equation (3).

When $g(t, x)=g(x)$, we can remove the assumption $\left(H_{2}\right)$ in Theorem 4.1 and obtain the following result.

Theorem 4.3. Assume that

$$
\left(H_{8}\right) 1-\sum_{i=1}^{m-1}\left|a_{2 i+1}\right|>0
$$

and the assumptions $\left(H_{1}\right),\left(H_{4}\right)$ are true. Then there exists at least one antiperiodic solution of equation $(3)(g(t, x)=g(x))$.

Proof. We consider the auxiliary equation of (3)

$$
\begin{aligned}
x^{(2 m+1)} & =-\lambda \sum_{i=2}^{2 m} a_{i} x^{(i)}-\lambda f(x) x^{\prime}-\lambda g(x)+\lambda p(t) \\
& :=\lambda q_{3}\left(x^{(2 m)}, \ldots, x^{\prime}, x, t\right)
\end{aligned}
$$

where $\lambda \in[0,1]$. Obviously, $q_{3}\left(x^{(2 m)}, \ldots, x^{\prime}, x, t\right)$ is continuous.

Similar with the proof of Theorem 3.1, we need only prove that there exists a prior bound in $C^{2 m+1, \pi}$, for the possible solution $x(t)$ of equation (11). 
Similar with the proof of Theorem 4.1, multiplying equation (11) with $x^{\prime}(t)$ and integrating it over $[0,2 \pi]$, we get

$$
\begin{aligned}
& \int_{0}^{2 \pi}\left|x^{(m+1)}(t)\right|^{2} d t \\
= & (-1)^{m+1} \lambda \sum_{i=1}^{m-1} a_{2 i+1} \int_{0}^{2 \pi} x^{(2 i+1)}(t) x^{\prime}(t) d t \\
& +(-1)^{m+1} \lambda \int_{0}^{2 \pi} f(x(t))\left(x^{\prime}(t)\right)^{2} d t \\
& +(-1)^{m+1} \lambda \int_{0}^{2 \pi} g(x(t)) x^{\prime}(t) d t+(-1)^{m} \lambda \int_{0}^{2 \pi} p(t) x^{\prime}(t) d t .
\end{aligned}
$$

Noting

$$
\int_{0}^{2 \pi} g(x(t)) x^{\prime}(t) d t=\int_{0}^{2 \pi} g(x(t)) d(x(t))=\int_{x(0)}^{x(2 \pi)} g(\tau) d \tau=0
$$

and by hypothesis $\left(H_{4}\right)$, we get

$$
\int_{0}^{2 \pi}\left|x^{(m+1)}(t)\right|^{2} d t \leq \sum_{i=1}^{m-1}\left|a_{2 i+1}\right| \int_{0}^{2 \pi}\left|x^{(i+1)}(t)\right|^{2} d t+\int_{0}^{2 \pi}\left|p(t) x^{\prime}(t)\right| d t .
$$

A similar argument with Theorem 3.1, we can prove that there exists at least one anti-periodic solution of equation $(3)(g(t, x)=g(x))$.

Similar with Theorem 4.3, we can obtain the following result.

Theorem 4.4. Assume that

$\left(H_{9}\right) 1-\sum_{i=1}^{m-1}\left|a_{2 i+1}\right|-M>0$

and the assumptions $\left(H_{1}\right),\left(H_{6}\right)$ are true. Then there exists at least one antiperiodic solution of equation $(3)(g(t, x)=g(x))$.

\section{References}

[1] A. R. Aftabizadeh, S. Aizicovici, and N. H. Pavel, On a class of second-order antiperiodic boundary value problems, J. Math. Anal. Appl. 171 (1992), no. 2, 301-320.

[2] T. Y. Chen, W. B. Liu, J. J. Zhang, and M. Y. Zhang, Existence of anti-periodic solutions for Liénard equations, J. Math. Study 40 (2007), no. 2, 187-195.

[3] F. Z. Cong, Q. D. Hunang, and S. Y. Shi, Existence and uniqueness of periodic solutions for $(2 n+1)$ th-order differential equations, J. Math. Anal. Appl. 241 (2000), no. 1, 1-9.

[4] K. Deimling, Nonlinear Functional Analysis, Springer-Verlag, Berlin, 1985.

[5] G. H. Hardy, J. E. Littlewood, and G. Pòlya, Inequalities, Cambridge University Press, 1952.

[6] B. W. Li and L. H. Huang, Existence of periodic solutions for nonlinear nth order ordinary differential equations, Acta Math. Sinica (Chin. Ser.) 47 (2004), no. 6, 11331140.

[7] W. G. Li, Periodic solutions for $2 k$ th order ordinary differential equations with resonance, J. Math. Anal. Appl. 259 (2001), no. 1, 157-167.

[8] W. B. Liu and Y. Li, Existence of periodic solutions for higher-order Duffing equations, Acta Math. Sinica (Chin. Ser.) 46 (2003), no. 1, 49-56. 
[9] J. Mawhin and M. Willem, Multiple solutions of the periodic boundary value problem for some forced pendulum-type equations, J. Differential Equations 52 (1984), no. 2, 264-287.

[10] M. Nakao, Existence of an anti-periodic solution for the quasilinear wave equation with viscosity, J. Math. Anal. Appl. 204 (1996), no. 3, 754-764.

[11] R. Ortega, Counting periodic solutions of the forced pendulum equation, Nonlinear Anal. 42 (2000), no. 6, Ser. A: Theory Methods, 1055-1062.

[12] M. A. Pinsky and A. A. Zevin, Oscillations of a pendulum with a periodically varying length and a model of swing, Internat. J. Non-Linear Mech. 34 (1999), no. 1, 105-109.

TAI YONG CHEN

Department of Mathematics

China University of Mining and Technology

Xuzhou Jiangsu 221008, P. R. China

E-mail address: taiyongchen@163.com

WEN BIN LiU

Department of Mathematics

China University of Mining and Technology

Xuzhou Jiangsu 221008, P. R. China

E-mail address: wblium@163.com

JIAN JUN ZHANG

Department of Mathematics

China University of Mining and Technology

Xuzhou Jiangsu 221008, P. R. China

E-mail address: zjj-cumt@163.com

Hui Xing Zhang

Department of Mathematics

China University of Mining and Technology

Xuzhou Jiangsu 221008, P. R. China

E-mail address: huixingzhangcumt@163.com 